\title{
Influence of Viewing Field on Zoom Levels in Pedestrian Orientation Task Using Smartphones
}

\author{
Bonan Wei ${ }^{a}$ *, Jochen Schiewe ${ }^{b}$ \\ a HafenCity University Hamburg, Germany, bonan.wei@hcu-hamburg.de \\ ${ }^{b}$ HafenCity University Hamburg, Germany, jochen.schiewe@hcu-hamburg.de \\ * Corresponding author
}

\begin{abstract}
Psychological findings indicate that the scale of human perception has implications on optimal map design. According to the map-based orientation in the real environment, this viewing scale depends on the visual field and is graphically reproduced using zoom levels, which significantly influences the map display area on smartphones. However, it is still unclear how to determine these zoom levels in the pedestrian navigation application. The purpose of this article is to adapt the map display area to the location-related viewing field using a corresponding zoom level. This optimal map display area should make it easier for the pedestrians' self-location and navigational decisions. The results of the experiments have shown that there was a close relationship between the viewing field and the zoom level on the smartphone. However, if the first decision point of changing direction was in the viewing field, the distance between the viewpoint (You-Are-Here point) and this decision point influenced the zoom level. Otherwise, this distance did not have any influence on the zoom level. In this case, the distance between the viewpoint and the local landmarks determined the zoom level.
\end{abstract}

Keywords: cognition of map design, pedestrian orientation, zoom level

\section{Introduction}

The first step of the map-based orientation task in a foreign environment begins with a matching process between perceived objects in the environment and the depiction of these objects on the map. This environment-map correspondence (Warren et al. 1990) is provided by the default view of a map navigation app, which will be displayed when opening an app at the starting point of the navigation. In the case of disorientation, the default view will be retrieved to determine the current location or to correct erroneous decisions. Based on the design principles of the You-Are-Here Map (Levine 1982) and the GNSS positioning technology, the default view is utilized, similar to Google Maps.

The architectonic and urban environment is a perceived space, which only exists for the observer and their perception (Joedicke 1985). This perceived space depends on the viewpoint of the environment and varies significantly from one place to another. The egocentric maps ensure that the ego (You-Are-Here point) is displayed in the geometric or optical center of the screen and the associated semantic contents are placed around this ego. Furthermore, this egocentric map is able to align the map depiction to the viewing direction. This kind of map is also defined as a forward-up egocentric map. It ensures that the perceived objects in the environment and the map depiction of these objects, in terms of the direction relation correspond with each other.

However, the map display area on a smartphone screen is not always correspondent to the viewing field at a given viewpoint. The reason for this lies therein that a default scale depending on the provider of the map app determi- nes the map display area. Moreover, the map display area depends on the size of the smartphone display. During the navigation of an urban environment, the visual field changes from one place to another and the spatial objects can be perceived from different distances. However, the content of the display area in combination with the zoom level remains constant and cannot be adapted to the viewing field. Due to the discrepancy between the locationrelated viewing field and the map display area, the map user must change the zoom level to obtain an optimal map to receive spatial orientation.

Adapting zoom levels to determine the map display area has long been implemented in vehicle navigation. Dillemuth and colleagues (2007) showed that map scales by six navigation systems are chosen arbitrarily. When creating an effective map interpretation, the scales can be determined by the parameters, such as speed, type of road, length of the route and interaction approach (Dillemuth et al. 2007). Wu and Zhang (2009) concluded that the scales depend on local roads and the decision points. Therefore, the scales should be adapted situationally for the task of navigation. Pedestrian navigation lacks knowledge in this issue in regarding to pedestrian orientation behavior (Kluge 2012). Several researcher investigated the effects of map display area regarding the acquisition of the spatial knowledge in the navigation task. Generally, the smaller the map display area, the less effective the map was in terms of accuracy, completion time (Dillemuth 2008) and distance estimation (Willis et al. 2009). Dillemuth (2008) noticed that the small map display area facilitated some aspects of acquisition of the spatial knowledge (e.g., turn recall). However, it was not clear as to how zoom levels in conjunction with the display area 
at a viewpoint could be adapted for an effective map interpretation and what factors influence the zoom level and how this happens.

In light of these considerations, the central research questions in this work are:

- How can the zoom levels in the mobile map be adapted in order to determine an optimal map display area size for the purpose of self-location?

- Which parameters of the viewing field correlate closely with the zoom level?

The aim is to identify the optimal map zoom level for pedestrians' self-location and navigational decisions. In chapter 2 , the question of how the map user perceives the environment for the purposes of self-location is discussed. Based on an interdisciplinary discussion, the central hypothesis is established that zoom levels correlate closely with the viewing field. In chapter 3 and 4, the three main influential factors on the zoom level are investigated in two experiments: (a) influence of the viewing field, (b) influence of global landmarks, (c) influence of the first decision point of the changing direction. In chapter 5, the unsolved questions are discussed.

\section{Literature Review and Hypotheses}

Lobben (2004) divided the matching process between perceived objects in the environment and the depiction of these objects on the map into two sub-processes: visualization (map-to-environment relation) and self-location (environment-to-map relation). The "act of seeing with the mind's eye, or developing a mental representation as a result of seeing a visual image, will be referred to as visualization. The term self-location refers to a person's ability to effectively relate the clues on the map to the represented real-word. Map readers solve the problem of determining their location on the map by recognizing real-world landmarks and relationships (clues), putting those clues together, and placing themselves on the map" (Lobben 2004). "If signs and labels seen on the map can be detected in the environment, relating features of the map to features of the environment is straightforward. Thus, self-localization as well as orientation and choosing the route become much simpler" (Richter and Klippel 2002).

Liben and Downs (1993) emphasized that the critical component in map-based orientation is to understand the self-map-space relation, which consists of three parts: self-map, map-space and self-space relation. To enhance the self-map and map-space relation, the related issues in cartography can be summarized mainly with three points of research:

- You-Are-Here point: The You-Are-Here point makes it possible to link one's location in the environment to one's location on a map of that environment. The You-Are-Here point facilitates the self-map relation.

- Map Alignment: The map display area can be aligned with the viewing direction. Therefore, the cognitive load effort induced by the requirement to mentally rotate maps can be minimized (McKenzie and Klippel 2016).

- Visualization of landmarks: The local landmarks as well as global landmarks have a decisive in- fluence on developing of the mental map (Siegel and White 1975) and determining of the direction at a decision point (May et al. 2003). The Map Alignment and Visualization of landmarks facilitate the map-space relation.

Self-space relation is an important research object in spatial cognition. However, the questions of how people perceive the space and how the perceived space relates to the self-location and navigational task have not yet received enough attention in the mobile map design.

The findings from spatial cognition provide fundamental knowledge that the scale element of human perception has an important influence on how humans treat spatial information (Montello 1993). The classification of the perceived spaces has implications for various theoretical and methodological questions concerning the design and use of spatial information tools (Freundschuh and Egenhofer 1997) such as the design of personal navigation systems.

On the basis of the projective size of the space relative to the human body, Montello (1993) distinguishes four classes of psychological spaces: figural, vista, environmental, and geographical space. Vista space is projectively as large or larger than the body which does not require locomotion to perceive it. For a self-location task, the variety of vista spaces needs to be taken into consideration, because only vista spaces are visible during navigation, never the whole environmental space (Meilinger et al. 2014).

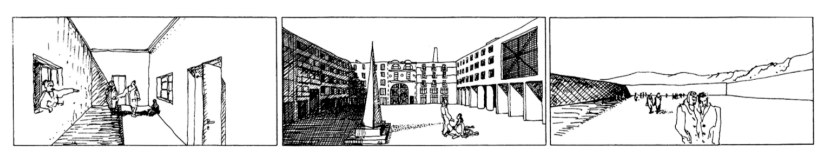

Figure 1. Vista space in three scales (Krier 1975)

The vista space can be classified in different scales (Figure 1). The successful self-location with the map (environment-to-map relation) is based on the congruence between perceived objects in the environment and the depiction of these objects on the map. In this context, the scales of vista spaces determine which information the map user needs to solve the self-location and navigational task and which section of the physical space should therefore be displayed. Based on the findings above, the central hypotheses can be proposed that zoom levels in the mobile map correlate closely with the different visual perceiving scales of the viewing field.

How do people perceive the objects in the viewing field and which parameters of the viewing field correlate closely with the zoom level? To answer these questions, the following hypotheses were established based on the following findings:

a) The zoom levels are influenced by the distance from a vantage point to the first decision point when changing directions: The importance of the decision points for the purpose of vehicle navigation can be found in the Wu and Zhang's (2009) and Ramos et al.'s (2016) experiments.

b) The zoom levels are influenced by the distances from a vantage point and the landmarks. It is indisputable that the landmarks are essential for naviga- 
tional tasks. Overall, there is a lot of research about the principles for selecting landmarks regarding orientation and about the strategies for using local or global landmarks to obtain a better navigational performance. However, there is disagreement as to which objects can be considered landmarks useful for map-based orientation. Some researchers underlined that landmark-based wayfinding cannot always be considered the most efficient navigation strategy (Montello 2017).

c) The zoom levels are influenced by the geometric shape of the viewing field (e.g., the area): The distance between the local landmarks and the viewing point in the viewing field represents the geometric layout and reflects the process of cognitive mapping. This geometric shape of the viewing field has an influence on the self-location (reorientation) (Meilinger 2007). Instead of using the local landmarks, the children can only orient themselves using the geometric layout (Hermer and Spelke 1994). The findings in architecture indicate that visual access (Weismann 1981) have a significant influence on spatial awareness. The geometric shape of an environment can also be represented by the parameters of the viewing field, such as area (Dillemuth 2005), roundness, jaggedness ${ }^{l}$ (Franz and Wiener 2008) and longest line of sight (Emo 2014).

\section{Experiment 1}

\subsection{Aim and Questions}

The overall purpose of this work was to predict the appropriate zoom level at any given viewpoint during the smartphone-based pedestrian orientation task. The aim of this experiment was to verify that the central hypothesis stating the close correlation between viewing field and zoom level. In this context, it was also indispensable to identify the influence of a global landmark at the zoom level, which can only be seen from a long distance. If a global landmark has a significant influence on the zoom level, it was assumed that there was no correlation between viewing field and zoom level. To achieve these aims, two questions were raised:

- Is there a close relationship between the zoom level and the viewing field?

- Does a global landmark have an influence on the zoom level?

\subsection{Defining Variables and Selection of Viewpoints}

An isovist is a polygon that captures spatial properties by describing the visible area from a given vantage point (Benedikt 1979). In ergonomics, the field of vision is the environment perceived by moving the head, but without moving the body (Kirchner and Baum 1990). In the following experiment, the visible area of a viewpoint was defined as the viewing field polygon, which was the combination of isovist and field of vision. The viewing field polygon facilitated a transformation from the cognitive representation of geometric parameters. Therefore, the independent variables were seven parameters of the viewing field polygon (Figure 2).

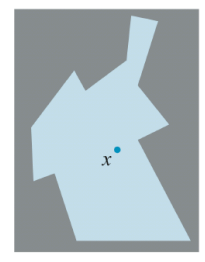

isovist-polygon at the viewpoint $x$

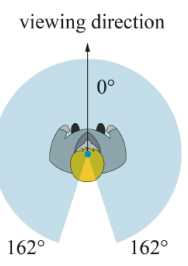

location-related visuel field at the viewpoint $x$

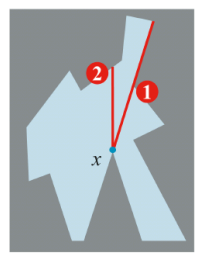

independent variable viewing field polygon

1. longest line of sight 2. line of sight
Figure 2. Concept of the viewing field polygon

The dependent variable was the zoom level decided by the map user, which is recorded using a tool written in Python. The map display area could also be changed using the functions of a graphical user interface, such as panning, tilting and rotating. Due to the limited scope of this work, the influence of those functions on the map display area was not discussed.

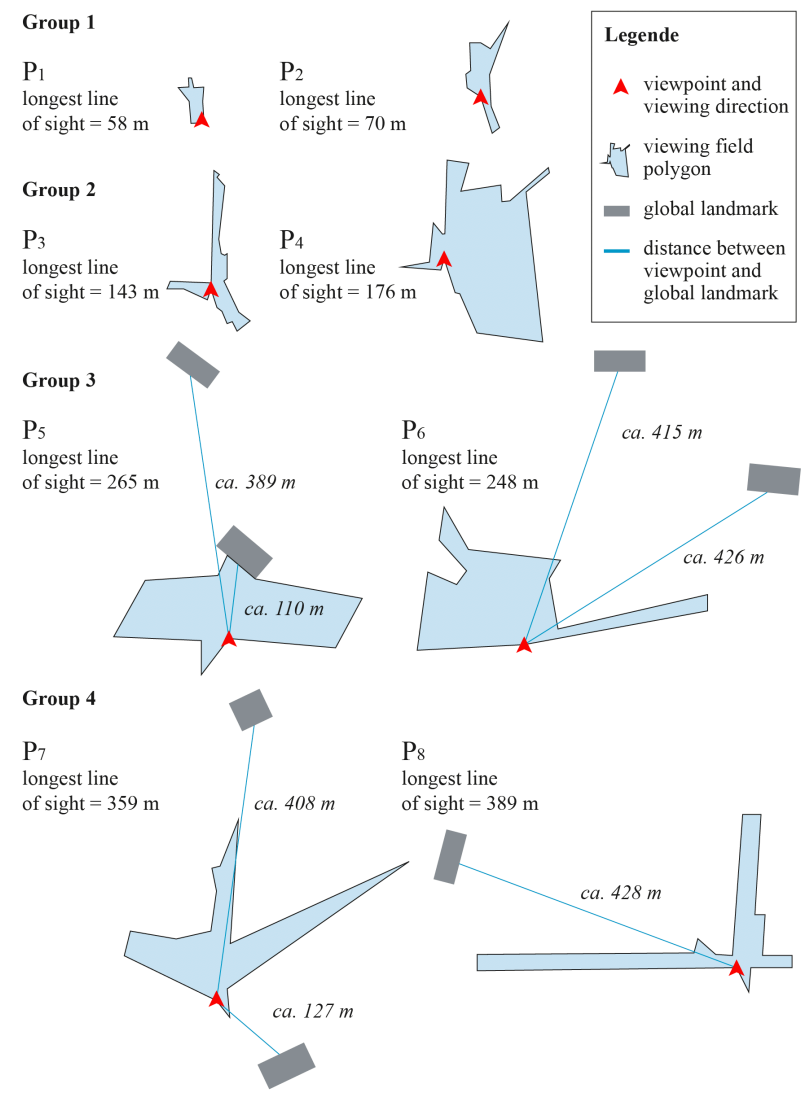

Figure 3. Selected viewpoints (Experiment I)

Eight viewing points were selected (Figure 3). Despite a relatively small number of viewpoints, it was possible to reflect spatial diversity. Figure 3 showed that two selected viewpoints in conjunction with the viewing field polygons had similar longest lines of sight and thus could be assigned to a group. Overall, eight viewpoints were formed into four groups. This grouping had the advantage in that the possible influence of the spatial features on the

\footnotetext{
1 Roundness (isovist area/perimeter ${ }^{2}$ ) and jaggedness (isovist perimeter $2 /$ area) describe the geometric layout of an architectonic environment in terms of the complexity (Franz and Wiener 2008).
} 
zoom levels could be reduced. If a viewing field polygon has a longest line of sight, it usually has a large area and a long perimeter. Therefore, the other parameters of the viewing field polygons were also considered. However, if the perimeter of a polygon is very long, it does not necessarily mean that the area of this polygon is very large. Such special cases were not investigated in this experiment.

Since the following experiment also aimed to identify the influence of global landmarks (e.g., church) on the zoom level, four viewpoints were selected so that these landmarks could be seen from different distances and directions from a given viewpoint.

\subsection{Research Design}

The user orientation behavior depends on visual perception, which is subjective and individual. For this reason, the statistical results may be insufficient for marking a well-founded scientific statement. In this context, the analysis of the qualitative data can be used to better understand the behavior and the thought processes of the participants. Therefore, this experiment was performed using methodological triangulation. This means that the data collection and analysis uses both a qualitative and quantitative approach.

The task was that each participant decided on a map display area using the zooming function within 30 seconds, by which the perceived surroundings could be better recognized for the purpose of self-location. The current location in the form of the You-Are-Here point was placed in the center of the screen. The tool written by Python was able to record the zoom level and map display area both at the end and during the working process. The starting zoom level of the default view was 17 (ca. scale 1:4.000). After choosing a map display area, the participants had to talk about which objects in the environment or on the map were important for this decision. Furthermore, the duration of the task completion was recorded. In the case of fast (duration $<10 \mathrm{sec}$.) or slow completion (duration $>30 \mathrm{sec}$.), the participants had to explain orally as to why the decision was made easily or difficulty. The purpose was to identify the potential influencing factors for the decision on the map display area.

The design of experiment I was a within-subjects design. 16 participants were recruited (female 12, male 4, average age 25 , body size ca. $165 \mathrm{~m}$ ). They had never been at these selected viewpoints before. Each participant had to complete the same tasks at 8 viewpoints under the same temporal and spatial conditions.

In total, 128 data sets were collected. This means that there were 16 map display areas selected by the same participants at each viewpoint. Hence, the variability of the collected data between the viewpoints has been minimized. In order to reduce the sequential confounding effect and order-effect, each of the two participants had an order of the viewpoints. Regarding the small number of participants, the orders of the viewpoints involved a partial counterbalance. This means that only eight orders have been taken into account. Moreover, it was ensured that each viewpoint appeared only once on a position in each order. Before the beginning of the experiment, a trial run was carried out.

\subsection{Interpretation of the Quantitative Data}

The analysis of the quantitative data consists of three parts: (1) the correlation analysis between the parameters of the viewing field polygon and the zoom level, (2) the analysis of variance between the means of the zoom levels at the different viewpoints, and (3) the analysis of variance between the means of the duration of the task completion at the different viewpoints.

The aim of the correlation analysis was to determine the relationship between the selected parameters of the viewing field polygon and the location-related zoom level. The Bravais-Pearson correlation analysis was performed because the data in terms of zoom levels fulfilled the conditions for the parametric tests. The results indicated that the zoom level was correlated with the parameters of the viewing field polygon (area $r=-0.67, r^{2}=0.45$; perimeter $r=-0.65, r^{2}=0.42$; the longest line of sight $r=-0.63$, $r^{2}=0.39$, line of sight $\left.r=-0.53, r^{2}=0.28\right)$. It was also apparent that there was a negative linear relationship between the zoom level and these four parameters. The coefficients of determination showed that the probability of the linear relationship was not very high. This could only be referred to as the mean and low variance explanation. Based on the correlation coefficient and coefficient of determination in terms of compactness $\left(r=0.40, r^{2}=0.16\right)$, roundness $\left(r=0.40, r^{2}=0.16\right)$ and jaggedness $(r=-0.40$, $r^{2}=0.16$ ), no explanation of variance could be demonstrated. The result confirmed the central hypothesis that the viewing field correlates closely with the zoom level for the purpose of map-based orientation using smartphones, e.g., the larger the viewing field, the smaller the zoom level should be. However, a high correlation between two variables does not mean that the two variables are causally related. Based on the relatively small number of selected viewpoints $(n=8)$, it could not be determined whether this relationship is exclusively linear. Moreover, the results show that most participants have only used the zoom levels between 16 and 19 .

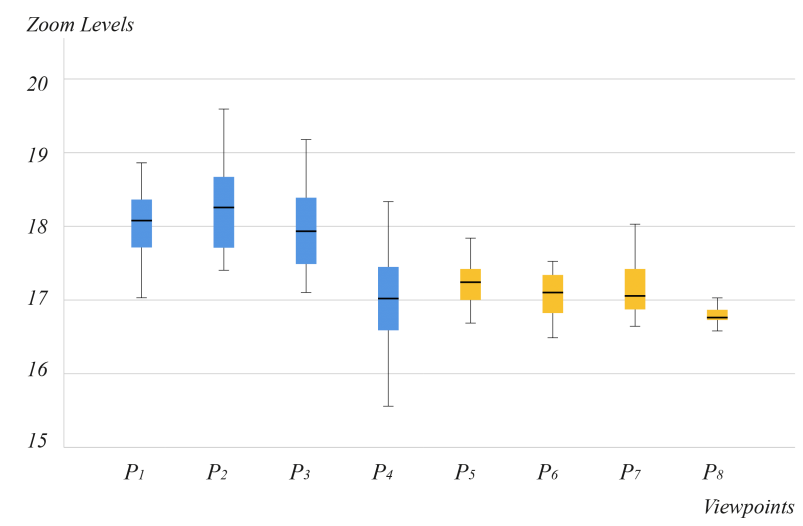

Figure 4. Box-Plot of ANOVA test without outliers (The blue boxes are the viewing field without global landmarks and the yellow boxes are the viewing field with global landmarks)

The aim of the ANOVA test was to determine whether the means of the zoom levels differed at eight selected viewpoints. Based on the results, the overall model was significant $\left(\mathrm{F}(7,105)=18.620, \mathrm{p}=0.000\right.$, partial $\left.\eta^{2}=0.554\right)$. The effect size $f=1.097$ corresponds to a strong effect. It could be formed by two well-differentiated groups regarding the zoom level (Figure 4): the zoom levels at viewpoints $P_{1}, P_{2}$ und $P_{3}$ did not differ significantly 
(mean=18.07) and the zoom levels at viewpoints $P_{4}, P_{5}$, $P_{6}, P_{7}$ and $P_{8}$ did not differ significantly (mean=17.04). This result indicated that it is not necessary to create an "autozoom". Two zoom levels should possibly be sufficient for map-based orientation using smartphones in an outdoor environment.

Moreover, based on this result, it was found that global landmarks had no influence on the zoom level. Despite the different direction and distance relations between viewpoint and the global landmarks, there was no difference in terms of the zoom level at viewpoints $P_{5}, P_{6}, P_{7}$ and $P_{8}$.

The purpose of the analysis of the duration of the task completion was to determine whether an inappropriate zoom level in conjunction with the corresponding map display area was the main cause of the long duration of map interpretation. The result indicated that the duration was not significantly different at 5 viewpoints $\left(P_{1}, P_{2}, P_{6}\right.$, $P_{7}$ and $\left.P_{8}\right)$. In comparison, all 16 participants needed only a very short time to decide on a map display area at viewpoints $P_{4}\left(\right.$ mean $=14 \mathrm{sec}$.) and $P_{5}($ mean $=10 \mathrm{sec}$.). At viewpoint $P_{4}$, the zoom level was slightly changed by the participants. At viewpoint $P_{5}$, the church was in the viewing field and could be directly perceived in the viewing direction. Furthermore, the church as a landmark was depicted in a different color displayed on the default view. This facilitated the creation of map-space correspondence. The longest time in terms of task completion was at viewpoint $P_{3}$ (mean=26 sec.), because there was no local landmark and all the houses looked almost the same. This result was surprising, because the zoom process was not the main reason for the long duration of the map interpretation. Although the participants had changed the zoom level from 17 to about 18 at viewpoints $P_{1}$ and $P_{2}$, they spent almost the same time as at viewpoints $P_{7}$ and $P_{8}$, where the zoom level did not need to be changed. An important finding was that local landmarks have a significant influence on effective map interpretation in terms of a short duration.

\subsection{Interpretation of the Qualitative Data}

The local landmarks recorded were represented using a thematic map and a directional diagram exemplarily. In the thematic map, it has been shown what kind of the objects as local landmarks the participants used and why the zoom level had to be changed. The road and the river as local landmarks were of high importance for mapbased orientation. This confirms Wrenger's (2015) findings, whereby the object of research in her study was the analogue map. The buildings at the road crossing or the single buildings in the environment were mostly used as local landmarks. This confirms the results in Janzen's (2000) studies. Janzen (2000) concluded that the objects at an intersection are frequently used as local landmarks. A house, which is located between two junctions or in a row of houses, is less important for spatial awareness. Subway stations were used as local landmarks because of their functionality and frequency (Hard et al. 1984). In summary, local landmarks are important for a map display area on a display. If an important local landmarks (e.g., bridge or road crossing) was not displayed, the map scale had to be zoomed out. If the local landmarks were at a shorter distance from the viewpoint and the distance relation between the viewpoint and the local landmarks depicted were illegible on the screen, the map scale had to be zoomed in.

The directional diagram focused on the local landmarks each participant individually used at the given viewpoints. The amount of local landmarks used by participants in order to establish the environment-map correspondence and in which order the local landmarks were used was shown. An average of 3.2 local landmarks at each viewpoint was used. These findings provided the evidence that two local landmarks as a minimum for the matching process between environment and map (Levine et al. 1982) are insufficient. Instead of two points localization, three points localization was necessary in this matching process. The distance relation is not only related to the distance between the viewpoint and a local landmark, but also to the distance between the different local landmarks. For example, some participants used the "reference area", such as the gap between two houses. Moreover, the objects in proximity to the viewpoint were often used as local landmarks, because the effort of mental rotation toward those objects was easier than with objects at a long distance.

Generally, both qualitative and quantitative statements proved the close relationship between the viewing field and the zoom level. The result of the correlation analysis indicated that the longest line of sight turned out to be one of the most important factors to identify the locationbased zoom level. The longer the longest line of sight, the smaller the scales will be. However, this finding did not necessarily correspond with the qualitative statements regarding the local landmarks recorded. Instead of the length of the longest line of sight, the distance between the viewpoint and the local landmarks located remotely is the key for determining the zoom level. In most cases, both lines of sight are congruent, but it was not without exception.

\section{Experiment II}

\subsection{Research Design}

The aim of experiment II was to identify the relationship between the first decision point for changing directions and the zoom level for the purpose of pedestrian orientation. The question was whether and to what extent the zoom level user needs at a given viewpoint could be influenced by the distance between the viewpoint and the first decision point when changing directions. Unlike the previous experiment, the experiments II was carried out with the between-subject design. Due to this, a counterbalancing procedure is not required. Like with experiment I, data collection was performed using methodological triangulation.

Five map display areas were made available for selection. The participants had to have seen all five map-sections at least once. Within a given time (30 sec.), each participant should select one of five map display areas so that they could better recognize their surroundings and could follow the route shown on the display. After this decision, they should explain which objects in the environment or on the map were of great importance for this decision. 
In contrast to previous experiments, the purpose was not to record the zoom level, because the map display area decided upon by participants can be influenced by the starting view (zoom level 17). In the previous experiment, several participants assumed that the starting display area was not the expected display area. Furthermore, the problem of the learning effect could arise if the participants observed the starting view for long time. In this experiment, the participants should decide without this bias on a map display area. Without a trial run, the warmup effect and the unnecessary proceedings of the zooming (e.g., test of the zoom function) cannot be minimized. In order to reduce the sequential order-effect, the orders of the map displayed maps were randomized.

Altogether, 72 participants were recruited for the 3 given points. All the participants had never been at this selected viewpoint before and used the same smartphone. There were 24 participants at each viewpoint. Depending on the distance between the viewpoint and the first decision point of the changing direction $(<50 \mathrm{~m}, 150 \mathrm{~m}$ and 250 $\mathrm{m})$, three groups of the 8 participants each were formed. Within a group at the given viewpoint, each participant had an identical viewing field. Due to the disadvantage of the between-subject design in terms of the differences between groups, the matched-groups procedure was be used. That means the participants in each group were recruited purposely, for example, using a distribution of the estimated average ages between 25 and 40 years and a distribution of gender in each group was almost equal.

\subsection{Interpretation of the Quantitative Data}

The aim of the correlation analysis was to determine the relationship between the area of the viewing field polygons and the zoom level. The analysis was performed by spearman's rank correlation, because the collected data in terms of the zoom levels did not meet the normality assumption for the parametric tests. The results indicated a negative linear relationship between the zoom level and the distance between the viewpoint and the first decision point of the changing direction $\left(r=-0.527, r^{2}=0.278\right.$, $p=0.000$ ). The shorter the distance between the viewpoint and the first decision point of the changing direction, the larger the zoom level should be. However, the zoom level was not correlated with the area of the viewing field polygon $\left(r=0.078, r^{2}=0.006, p=0.515\right)$. Furthermore, the result of the Kruskal-Wallis Test confirmed that the zoom level would be larger if the distance between the viewpoint and the first decision point of the changing direction is short $\left(\chi^{2}=21.082, \mathrm{p}=0.000\right)$. The Dunn-Bonferroni tests showed that group I $(<50 \mathrm{~m})$ differed from group II $(150 \mathrm{~m}, \mathrm{z}=3.242, \mathrm{p}=.004, \mathrm{r}=0.468)$ and group III $(250$ $\mathrm{m}, \mathrm{z}=4.437, \mathrm{p}=0.000, \mathrm{r}=0.640)$ significantly. It indicated that the distance between the viewpoint and the first decision point of the changing direction had an influence on the zoom level. However, there was no significant difference between group I (150 m) and group II (250 m) $(\mathrm{p}=0.697)$. This means that the distance between the viewpoint and the first decision point of the changing direction did not have any influence on the zoom level, if it was not in the viewing field or was rather very distant from the viewpoint (e.g., $250 \mathrm{~m}$ in this experiment).

\subsection{Interpretation of the Qualitative Data}

The findings of the evaluation of the qualitative data refuted the result of the evaluation of the quantitative data in that the distance between the viewpoint and the first decision point of the changing direction had a significant influence on the map display area. Based on the recorded local landmarks, which the participants used in the matching process between the map and the space, it could be determined that the first decision point in the walking direction was critical to selecting the map display area for spatial orientation (e.g., the first crossing in the walking direction). This is independent of whether the participants had to change direction at this point.

Contrary to the self-location process, most participants saw the map depiction at first. In the map-spacematching process, the participants attempted to recognize the depicted route in the environment (e.g., street). The local landmarks the participants used were related with this decision point. For example, the walkway is between two houses (local landmarks). Due to this, it was established that the space perception during the map-space matching process was carried out by the three points localization. This distance relation between viewpoint and local landmarks have to be better represented on the map display area on the smartphone in order to ensure a better map interpretation.

This finding provided an important insight in that the first decision point in the walking direction and the spatially related local landmarks in the viewing field have a significant influence on the location-related zoom level. However, if the first decision point of the changing direction is in the viewing field, it has a significant influence on the zoom level. This distance relation did not have any influence on the zoom level if the first decision point of the changing direction was not in the viewing field or rather very distant from the You-Are-Here point. In this case, the zoom level was determined by the parameters of the viewing field and by the distance between the viewpoint and the first decision point in the walking direction (Figure 5).
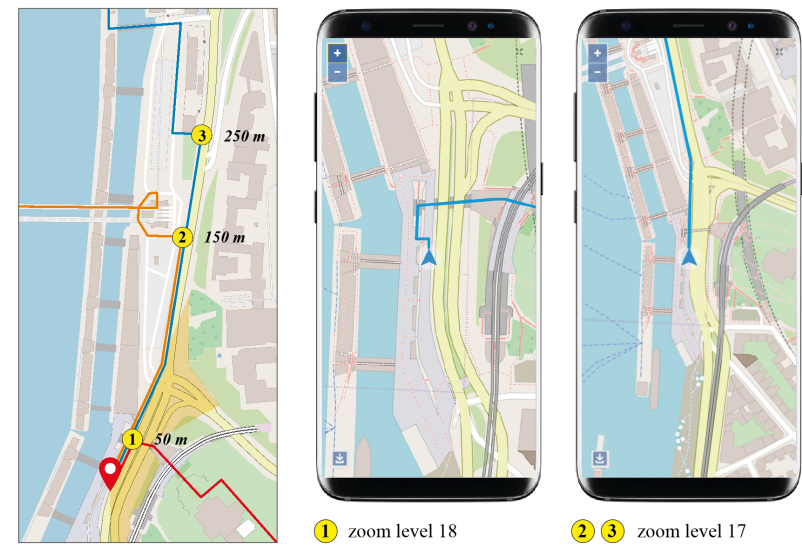

(2) (3) zoom level 17

Figure 5: Example at the viewpoint 10 (left: three decision points of the changing direction; middle: most selected map display area in the group I; right: most selected map display area in the group II and III).

\section{Discussion}

Method: The test procedure in the real world can be considered appropriate for the research purpose, even though there were more interfering factors and the test conditions 
could not be more effectively controlled compared to laboratory conditions. The reason for this lies therein that the research that uses the figural spaces (e.g. photos or videos displayed on the computer screen as stimuli) to study the vista space may be questionable (Montello 1993).

Concept of Viewing Field Polygon: Generally, the viewing field polygon could be regarded as an appropriate concept for the purpose of this research. However, this geometric transformation is only one solution for the representation of the user-perceived visual environment map and has some open questions:

a) Cutting (1997) and Golledge (1999) pointed out that cognitive representation need not bear a one-toone correspondence with their counterparts in physical space. Future studies should explore whether the distortion of the viewing field polygon in the vista space have influences the zoom level.

b) The objects in the environment, which are more than five meters in height, were considered to be an obstacle in the previous experiments. In this context, two questions arise as to whether this determination of the height as expedient for the representation of the viewing field at a given viewpoint and which objects in the environment should be regarded as obstacles. On the one hand, the height of the objects is an important factor, while on the other hand, the transparency of the objects are also essential in the discussion. Based on this concept, a big tree could be considered as obstacle, because the objects behind the trees may not be perceived visually. However, this may only be considered during certain seasons (e.g., in summer). As a result, the viewing field polygons must be changed accordingly in winter, because the objects behind the trees may be perceived visually and should therefore no longer be considered obstacles.

c) The changing of the viewing field polygon must be considered regarding weather conditions and time of day.

Urban Typology: "The way people conceptualize space is an important consideration for the design of geographic information system" (Freundschuh and Egenhofer 1997). Although Krier (1975) argued that the scales do not affect the arrangement of the typology, it is nonetheless important to investigate the influence of different types of urban spaces on the same scale in connection with the different architectural conceptions on the zoom level.

\section{Outlook}

Positioning of You-Are-Here Point on a Screen: In the research area of the egocentric map design, it is determined that the You-Are-Here point should be positioned at the geometric or optical center of the screen. Multiple research underlines that the position of the You-Are-Here point should not be changed during navigation. If both the display area and the You-Are-Here point on the screen were changed, the effort of the mental rotation would be greater (Kim et al. 1997). Winter and Tomko (2004) underline that it is more intuitive for a map user to find their actual position at the bottom of the map. The qualitative data analysis indicated that most local landmarks used by the participants were located in the direction of viewing. If there are local landmarks (e.g., subway station) behind the map user, it could also be used as a local landmark. In the case that a map user orients himself after exiting the building, this building may be used as a local landmark because the place showed where the user came from. Therefore, it is not appropriate that the You-Are-Here point is placed at the bottom of the screen for the purpose of pedestrian orientation. Perhaps it should be positioned on the vertical centerline of the screen, with more space on the top of the display and less space from the bottom. However, this assumption should be determined empirically in future works.

Map Depiction and Labels: In these experiments, the influence on the labels and map depiction were not taken into consideration. In this context, the question must be raised as to which labels should be displayed on the different zoom levels for the purpose of spatial orientation. Furthermore, the question is important as to what extent the local landmarks on the different zoom levels should be generalized.

Uncertainty of the Viewing Field Polygon: Based on the results, it was found that the difference of the zoom level that the participants needed at several viewpoints was not significant, although the distinction in terms of the parameters viewing field polygons could be very large. Therefore, it can be assumed that the deviation between the GNSS localized You-Are-Here point and the current viewpoint does not have any influence on the locationrelated zoom level. Furthermore, the uncertainty in terms of the drawing of the viewing field polygon could also be tolerated. However, this assumption was based on a viewpoint in these experiments in an outdoor environment. It is likely that the zoom level may be influenced by the uncertainty of the viewing field polygon in an indoor environment.

Influence of Screen Size on Zoom Levels: In an on-going experiment, a two-way ANOVA test with repeated measures will be used to identify whether and to what extent smartphone diagonals have an influence on the zoom level at a given viewpoint. The experiment is carried out at four viewpoints in conjunction with four different viewing field polygons. Each viewing field polygon is distinguished from others because of the parameter area. The questions are:

- do the smartphone diagonals (4 and 6.2 " inch) have a significant influence on the locationbased zoom level map-users need?

- Is there a statistically significant interaction between a smartphone diagonal and the area of the viewing field polygon?

Multiple Windows on a Smartphone Display: Tversky (2000) categorized mental representations of three levels: Overview, View and Action. For each level, map users expect different information at each scale. During navigation, map users need the display area for decision making in terms of the environment-map correspondence as well as the overview of the route. For this purpose, the design of multiple windows on the screen could be considered a possible solution. In this experiment, the maximal map extension the participants have seen were determined using the tracking function. In most cases, these maximal 
map extensions were larger than the displayed area the participants finally decided upon. However, it is unclear as to what relationships exists between this maximal map extension and the display area.

This practice-oriented explorative study provides new research findings with regard to the connection between the scale of the perceptual space and the scale in mobile map depiction for the purpose of a pedestrian self-location and navigational task. Furthermore, this finding represents a major step towards the goal of achieving the User-Centered-Design of pedestrian map apps. To take this thought one step further, it may well be that these research findings could contribute to seamless pedestrian navigation between indoor and outdoor environments. Beyond pedestrian navigation, this research also has the potential to gain practical significance for other navigation tasks (e.g., for creating a fire escape plan).

\section{References}

Benedikt, M. L. (1979). To Take Hold of Space: Isovists and Isovist Fields. Environment and Planning B6, pp. 47-65.

Cutting, J. E. (1997): How the eye measures reality and virtual reality. Behavior Research Methods, Instruments, \& Computers 29, Nr. 1, pp. 27-36.

Dillemuth, J.A. (2005). Map Design Evaluation for Mobile Display. Cartography and Geographic Information Science 32(4), pp. 285-301.

Dillemuth, J.A., K. Goldsberry \& K.C. Clarke (2007). Map Design Evaluation for Mobile Display. Journal of Locational Based Services 1(1), pp. 46-61.

Dillemuth, J.A. (2008). Map Use and Spatial Knowledge Acquisition with Small, Mobile Map Displays. Dissertation, University of California.

Emo, B. (2014). Real-World Wayfinding Experiments, Individual Preferences, Decisions and the Space Syntax Approach at Street Corners. Dissertation, University College London.

Franz, G. \& Wiener, J.M. (2008). From Space Syntax to Space Semantics: A Behaviorally and Perceptually Oriented Methodology for the Efficient Description of the Geometry and Topology of Environments. Environment and Planning B: Planning and Design 35(4), pp. 574 592.

Freundschuh, S.M. \& Egenhofer, M.J. (1997). Human Conception of Spaces. Implications for Geographic Information Systems. Transactions in GIS 2(4), pp. 361375.

Golledge, R. D. (1999): Human Wayfinding and Cognitive Maps. In: Wayfinding Behavior, John Hopkins Press, pp. 5-45.

Hard, G., Jessen, F. \& Schirge, M. (1984). Umweltwahrnehmung in der Stadt - Eine Hypothesensammlung und eine Empirische Studie. In H. Köck (Eds.), Studien zum Erkenntnisprozess im Geographieunterricht, Köln: Aulis-Verlag Deubner, pp. 113-165.

Hermer, L. \& Spelke, E.S. (1994). A Geometric Process of Spatial Reorientation in Young Children. Nature 370 (6484), pp. 57-59.
Janzen, G. (2000). Organisation räumlichen Wissens: Untersuchungen zur Orts- und Richtungsrepräsentation. Wiesbaden: Springer Fachmedien.

Joedicke, J. (1985). Space and Form in Architecture. A Circumspect Approach to the Past. Stuttgart: Karl Krämer Verlag.

Kim, B., Han, S.H., Nam, K., Park, J. \& Han, S. (1997): Presenting Map Information on a Global Positioning System. Computers and Industrial Engineering 33 (3-4), pp. 529-532.

Kirchner, J.H. \& Baum, E. (1990). Ergonomie für Konstrukteure und Arbeitsgestalter. Munich: Carl Hanser Verlag.

Kluge, M. (2012). Der Einsatz von Augmented Reality in der Fußgängernavigation. Konzeption und prototypische Implementierung eines smartphonebasierten Fußgängernavigationssystems. Dissertation, University Potsdam.

Krier, R. (1975). Urban Space. Solingen: Umbau Verlag.

Levine, M. (1982). You-Are-Here maps. Psychological Considerations. Environment and Behavior 14, pp. 221237.

Levine, M., Jankovic, I.N. \& Palij, M. (1982): Principles of Spatial Problem Solving. Journal of Experimental Psychology General 111(2), pp. 157-175.

Liben, L.S. \& Downs, R.M. (1993). Understanding Person-Space-Map Relations: Cartographic and Developmental Perspectives. Developmental Psychology 29 (4), pp. 739-752.

Lobben, A.K. (2004). Tasks, Strategies and Cognitive Processes Associated With Navigational Map Reading: A Review Perspective. The Professional Geographer 56(2), pp. 270-281.

May, A.J., Ross, T., Bayer, S.H. \& Tarkiainen, M.J. (2003). Pedestrian Navigation Aids: Information $\mathrm{Re}^{-}$ quirements and Design Implications. In: Personal and Ubiquitous Computing, Issue 6, Bd. 7, London: Springer, pp. 331-338.

McKenzie, G. \& Klippel, A. (2016). The Interaction of Landmarks and Map Alignment in You-Are-Here Maps. The Cartographic Journal 53(1), pp. 43-54.

Meilinger, T. (2007). Strategies of Orientation in Environmental Spaces. Dissertation, Albert-Ludwigs-University Freiburg.

Meilinger, T., Riecke, B. E. \& Bülthoff, H. H. (2014): Local and Global Reference Frames for Environmental Spaces. Quarterly Journal of Experimental Psychology 67(3), pp. 542-569.

Montello, D.R. (1993). Scale and Multiple Psychologies of Space. In A. U. Frank and I. Campari (Eds.), Spatial Information Theory. A Theoretical Basis for GIS, Lecture Notes in Computer Science, Bd. 716, Berlin and Heidelberg: Springer, pp. 312-321.

Montello, D.R. (2017). Landmarks are Exaggerated. Künstliche Intelligenz 31(2). Springer, pp. 193-197.

Ramos, A. P. M., Decanini, M. M. S., Pugliesi, E. A., Tachibana, V. M., De Oliveira, R. F. \& Soares, J. M. M. (2016): Preference for Map Scale of In-Car Route Guidance and Navigation System. Boletim de Ciências Geodésicas 22(3), pp. 472-491. 
Richter, K.F. \& Klippel, A. (2002). You-Are-Here Maps: Wayfinding Support as Location Based Service. In J. Moltgen and A. Wytzik (Eds.), GI-Technologien für Verkehr und Logistik Beiträge $\mathrm{zu}$ den Münsteraner GITagen, pp. 363-382.

Siegel, A. \& White, S. (1975). The Development of Spatial Representations of Large-scale Environments. In: H. W. Reese (Eds.), Advances in Child Development and Behavior, Bd. 10, New York: Academic Press, pp. 1055 .

Tversky, B. (2000): Levels and Structure of Spatial Knowledge. In: R. Kitchin und S. Freundschuh (Eds.), Cognitive Mapping: Past, Present and Future. New York, London: Routledge, pp. 24-43.

Warren, D.H., Rossano, M.J. \& Wear, T.D. (1990). Perception of Map-Enviornment Correspondece: The Roles of Features and Alignment. Ecological Psychology 2(2), pp. 131-150.

Weisman, J. (1981). Evaluating Architectural Legibility Wayfinding in the Built Environment. Environment and Behavior 13(2), pp. 189-204.

Willis, K.S., Hölscher, C., Wilbertz, G. \& Li, C. (2009). A Comparison of Spatial Knowledge Acquisition with Maps and Mobile Maps. Computers, Environment and Urban Systems 33(2), pp. 100-110.

Winter, S. \& Tomko, M. (2004). Shifting the Focus in Mobile Maps. In Morita, T. (Eds.), Joint Workshop on Ubiquitous, Pervasive and Internet Mapping [online]. URL: https://people.eng.unimelb.edu.au/winter/pubs/ winter04shifting.pdf [29.11.2018]

Wrenger, K. (2015). Kartengestützte Orientierung im Realraum unter besonderer Berücksichtigung der Einflussgröße Raum. Dissertation, University Koblenz.

Wu, A. \& Zhang, X. (2009). Identifying Proper Scales on Digital Maps for In-Vehicle Navigation Systems. In Proceedings of the 5th International Conference on Universal Access in Human-Computer Interaction. Lecture Notes In Computer Science, vol. 5616. Berlin, Heidelberg: Springer, pp. 262-270. 